\title{
Strategic Informative Advertising in a Horizontally Differentiated Duopoly*
}

\author{
Levent Çelik ${ }^{\dagger}$
}

October 2006

\begin{abstract}
Consider a horizontally differentiated duopoly market where potential buyers are uncertain about their matches with either product. Would informative advertising by a firm about its own product disclose any information about the other product when firms know how their as well as their rival's product matches buyers' preferences? I answer this question in the context of a television (TV) market that lasts for two periods in which viewers are uncertain about the attributes of the upcoming programs. A symmetric perfect Bayesian equilibrium (PBE) in which advertising decisions of the TV stations depend on the attributes of both programs exists, and is the only such strategic equilibrium. Although not fully revealing, it enables viewers to narrow down their priors. When this PBE is unattainable, the only other equilibrium is one in which the advertising decision of a station is independent of the other program's attributes. While it is welfare improving to ban such advertising if the latter PBE arises as the market outcome, this is not necessarily true for the former one. This raises an obvious empirical question: Do TV stations act strategically while advertising their own programs?
\end{abstract}

Keywords: Informative Advertising, Tune-ins, Uncertainty, Sampling, Signaling. JEL Classification: D83, L13, L82, M37

\footnotetext{
${ }^{*}$ I would like to thank my dissertation advisors at the University of Virginia, Simon Anderson and Maxim Engers, for their invaluable help during the progress of this paper.

${ }^{\dagger}$ CERGE-EI (a joint workplace of the Center for Economic Research and Graduate Education, Charles University, and the Economics Institute of the Academy of Sciences of the Czech Republic), Politickych Veznu 7, 111 21, Praha 1, Czech Republic. Email: Levent.Celik@cerge-ei.cz.
} 


\section{Introduction}

There is a broad range of consumer markets where the differentiation among products is mainly in their physical attributes. Although the information about the existence of these products may be common knowledge, many people may have limited or even no information about their attributes. This lack of information may be due to the fact that the products are newly introduced, or that the costs associated with gaining information are relatively high. Television (TV) industry is a good example. It is almost impossible for people to know the attributes of all of the programs at different TV stations. Gaining accurate information through TV guides or internet is often costly. Furthermore, individuals have limited memories. The present paper proposes a two-period model of informative advertising in a TV market with two TV stations when potential viewers are uncertain about their match values with the programs in the latter period. A crucial element of the model is that the TV stations know how their own as well as their rival's program fits viewers' preferences. It is shown that this information structure leads to an equilibrium in which the decision of a TV station to advertise its own program may strategically depend on its rival's program, and viewers correctly anticipate this strategic interaction.

The use of advertising has been increasingly rising in recent years. Total U.S. spending on advertising in 2003 had been recorded as $\$ 245.5$ billion. Approximately $25 \%$ of this amount had been devoted to TV commercials at network and cable TV stations (network: 17.1\%, cable: $7.7 \%$ ). A company had to pay over $\$ 600,000$ for a 30 -second commercial during the popular show "American Idol" in 2004. Three major broadcast stations, CBS, ABC and NBC, reported advertising revenues over $\$ 5$ billion for the year 2003. The price of a 30second commercial during Super Bowl has been over $\$ 2$ million since 1999 (\$2.6 million in 2006), yet the TV stations airing the event have continued to devote approximately $20 \%$ of the non-program time to tune-ins (preview advertisements of their own programs). During 2000 Super Bowl, 16.5 of a total of 87 non-program minutes were used for tune-ins. These simple statistics reflect the importance of tune-ins for TV stations. Had viewers already been fully informed about the attributes of these programs, there would be no need for tune-ins. 
Çelik (2006) takes a look at the extent to which a single TV station is willing to air a tunein. The model is developed in a simple Hotelling framework in which there is a continuum of potential viewers distinguished by their ideal programs. This is represented by assigning a unique location to each potential viewer along the unit line. As usual in Hotelling models, a viewer's net utility is lower the further away the actual program is from her ideal program. There is a single TV station that airs two consecutive programs. The location of the first program is assumed to be common knowledge. This may be thought of as the evening news program. The location of the second program is ex-ante unknown by viewers. However, the viewers know that the TV station is privately informed about its location. Therefore, they rationally anticipate that the TV station would communicate this piece of information with the first-period audience unless it is worse off by doing so. The cost of airing a tune-in is the forgone revenue from a commercial advertisement during the first program. In this setting, it is shown that there exists a unique perfect Bayesian equilibrium (PBE) in which the TV station airs a tune-in as long as the advertising revenue generated by the viewers continuing to watch offsets or exceeds the cost of airing it. In the absence of a tune-in, no one from among the first-period audience keeps watching TV.

In order to extend the analysis above to include a second station, one has to introduce the possibility of switching from one station to the other. In fact, Celik (2006) introduces as an extension the possibility of switching off after sampling a few minutes of a program. While a PBE similar to the one described above remains to exist, it is no longer unique. For certain parameter values, there exists another PBE in which the TV station never airs a tune-in. In this paper, I move one step further by incorporating viewers' switching behavior into the same setup when there are two TV stations. I assume that the amount of time required for learning the actual location of a program is fixed and the same for all programs and individuals. However, this process entails an opportunity cost if an individual does not continue to watch the program she chose to sample.

The process of costly sampling plays a crucial role for two reasons. First, for an equilibrium that involves the use of tune-ins to exist, sampling cost (or equally switching cost) has 
to be positive. Had it been zero, viewers could costlessly learn the programs at both stations and make their decisions without any uncertainty. Therefore, there would be no need for tune-ins. Second, a positive sampling cost may create an incentive for a station to choose not to air a tune-in. This is because the cost of sampling becomes sunk once a viewer chooses to engage in sampling. That is, when there is costly sampling, some individuals may end up watching a program that they would not choose to watch with complete information. By the same token, an individual's final decision may not be the one that maximizes her utility with complete information. That a positive sampling cost is necessary for the existence of tune-ins is empirically confirmed by the statistics given earlier. It is harder to establish empirical support for the second one because of limited individual-level data. However, Shachar and Emerson (2000) report that $65 \%$ of viewers continue to watch the same network station, including the times when a tune-in has not been aired.

When the TV stations are informed about each other's program content, their decision to air a tune-in may transmit information about not only their own program but also their rival's. Since sampling is costly, a station is relatively more inclined to air a tune-in in order to lock-in its current viewers when its rival has a more similar program. However, this may signal to the recipients of that tune-in that the program at the other station is more likely to be a good match than what they thought before. Similarly, if a station does not advertise its upcoming program, it does not necessarily mean that it is a bad match for that station's viewers. It could rather be the case that it is a better match than the other program. In this paper, I am primarily interested in exploring the nature of such strategic behavior, and ultimately in finding out if an equilibrium in which viewers' priors are changed at an interim stage exists. I show that such an equilibrium exists although it is not unique. Without any restriction on viewers' beliefs, there is another equilibrium in which viewers' beliefs about either program are unchanged regardless of the tune-in decisions of the stations.

Signaling has traditionally been investigated within the context of vertically differentiated products. When consumers are uninformed about the actual quality of an experience good, it has been shown that a high-quality seller can credibly signal this information by setting 
a high price or by spending a non-trivial amount of money on uninformative advertising. My findings suggest that signaling is also possible in horizontally differentiated markets. Signaling occurs whether a station chooses to or not to air a tune-in. In the former case, only information about the other program is signalled. In the latter, information about both programs is signalled. However, a fully separating equilibrium is not possible in the current setting; viewers cannot locate the programs with certainty.

For certain program locations, the model can also be interpreted as one with vertical differentiation. To be more specific, when a station's upcoming program is better suited to all of its current viewers than the other station's upcoming program, the two programs are effectively vertically differentiated for those viewers. In such a situation, I find that the former station does not air a tune-in. Although this result is strikingly different than what traditional models of signaling predict, a direct comparison may be misleading since TV programs are not experience goods. Neverthless, it is interesting to note that signaling is possible even in the absence of advertising.

I also analyze the welfare effects of a possible ban on the use of tune-ins. I find that when it is not a credible strategy for a station to behave strategically, it may be welfare improving to ban tune-ins. In such a situation, the stations advertise their programs more often. Although viewers enjoy a higher surplus as a result of improved information, social welfare is reduced because the decrease in revenues of the TV stations overweights the increase in consumer surplus.

\section{Previous Literature}

Directly informative advertising has been the topic of several previous studies. Butters (1977) was the first to model the informative role of advertising. In his paper, products are homogeneous and advertising conveys information about prices, hence also about the existence of the products indirectly. However, much advertising involves informing consumers about product attributes other than just about prices. Grossman and Shapiro (1984) study an extended model in which consumers are heterogeneous in their preferences and advertising 
informs them not only about the existence but also about the characteristics of the products. Common to both of these papers is that the advertising technology is exogenous and people cannot change their likelihood of receiving an ad.

The current paper has several similarities with the latter of the mentioned papers; consumers are heterogeneous in their preferences and seek to purchase the product yielding the highest (expected) benefit, products are horizontally differentiated, and advertising provides information about product attributes. I assume that it is free to watch TV, and that the number of total non-program breaks is given exogenously. Therefore, my analysis does not involve price advertising. However, I depart from Grossman and Shapiro (1984) in several ways, mainly in how advertising is modeled. Advertising in this paper is exclusive; only the viewers who watch the first program may receive a tune-in of the second program at that station. It is also strategic in the sense that a station's decision to air or not to air a tune-in conveys information about the programs at both stations.

Confining attention to the literature that focuses on informative advertising in an oligopoly market with horizontally differentiated products, a related paper is Meurer and Stahl (1994). They analyze the welfare properties of informative advertising in a duopoly where a fraction of buyers are uninformed about the product characteristics. There are two types of buyers. One type is ideally matched with one firm and the other type is ideally matched with the other firm. As in Butters (1977), Grossman and Shapiro (1984) and many others, a firm chooses its advertising intensity and a random fraction of consumers receive the ad. Advertising informs a buyer of her best match. Firms choose their prices after advertising takes place. They treat product information as a public good, which implies that information about one product provides information about the others as well. They characterize a unique subgame perfect Nash equilibrium in which the level of advertising provided may be more or less than socially optimal. While advertising improves the match between consumers and products, it gives firms a higher market power by increasing brand loyalty.

Within the same strand of literature, another related paper is Anand and Shachar (2006). They use the same setup with that of Meurer and Stahl (1994) with three major differences. 
First, a firm can only advertise through one or both of the two available media channels, and consumer preferences over product attributes are perfectly correlated with their choice of media channel. So, for instance, if consumers of media channel 1 are ideally matched with product 1 , then firm 1 can target these consumers by advertising through media channel 1 . Second, advertising messages are noisy in the sense that consumers may get the wrong idea from a firm's informative ad. Therefore, firms advertise more than once. Finally, firms do not choose prices, which are therefore suppressed in the analysis. In such a setting, Anand and Shachar (2006) characterize a separating equilibrium in which a firm advertises only to those consumers for whom that product is the ideal one. As long as the ads are not completely noisy - in which case the ads would equally be interpreted right or wrong - there exists a threshold amount of advertising which ascertains a consumer that the advertised product is her best match. Thus, regardless of the content of the ad, each consumer purchases the product that she was advertised to.

There are major differences between my model and those of Meurer and Stahl (1994) and Anand and Shachar (2006). First, in both of these papers, products are experience goods, so consumers do not have the option of obtaining product information by a costly search. In the current paper, I treat TV programs as search goods since program sampling is a common practice in real life. If I rather treated them as experience goods, the unique symmetric equilibrium would involve no strategic behaving by the stations. Therefore, sampling plays a crucial role for the results in this paper. Second, there are only two distinct types of consumers in both papers, one ideally matched with one product and the other with the other product. In Meurer and Stahl (1994), this assumption implies that an informative ad by one firm necessarily informs the recipient about the other firm's product, and therefore plays a critical role for their results. In Anand and Shachar (2006), it is a necessary assumption for perfect separation. In my model, on the other side, there is a continuum of people who may or may not be ideally matched with either program. Therefore, the tune-in decision of a station is a function of the program location of the other station. Third, advertising in my model is purely informative unlike as in Anand and Shachar (2006), and reaches a 
nonrandom group of consumers unlike as in Meurer and Stahl (1994). In this sense, I use a different advertising technology in the current paper.

The current paper is also weakly related to the literature on quality signaling. To the best of my knowledge, the only two papers that address quality signaling when firms have common knowledge of product qualities are Matthews \& Fertig (1990) and de Bijl (1997). In Matthews \& Fertig (1990), this is introduced in the context of an incumbent-entrant setup where product quality of the entrant is known to both firms while that of the incumbent is common knowledge. Prices are exogenous and the incumbent may advertise in order to inform consumers about the product quality of the entrant; i.e. may counteract misleading attempts by a low-quality entrant. In sharp contrast to the existing models of quality signaling, they show that a high-quality entrant can successfully signal its quality by spending an infinitesimal amount on advertising. De Bijl (1997) analyzes entry-deterrence in a market for search goods when product quality of the entrant is known to both firms while that of the incumbent is already established. It is shown that when the incumbent's price is informative about the product quality of the entrant, entry of a high-quality entrant is facilitated. ${ }^{1}$

\section{The Model}

The basic setup is that of Celik (2006) with the exception that there are now two TV stations, station $Y$ and station $Z$, each airing two programs in two consecutive time periods. The programs are characterized by their locations on the unit line. They are of the same length and have zero production costs. There are $A$ available non-program breaks during each program in each period. ${ }^{2}$ There is a large number of firms that are willing to pay up

\footnotetext{
${ }^{1}$ See also Hertzendorf \& Overgaard (2002) who analyze signaling with price-only when firms have common knowledge about product qualities.

${ }^{2}$ The assumption that the number of non-program breaks is fixed is certainly restrictive. However, while U.S. broadcasters are free to choose the number of their commercial breaks, advertising ceilings are imposed on broadcasters in most European countries. Therefore, in most cases, especially in the prime time, the number of commercial breaks that maximize a broadcaster's revenues falls below the imposed ceiling. I believe that this empirical fact constitutes a good justification. Aside from the empirical side, there are technical reasons for this assumption. First, if TV stations were allowed to choose the number of non-program breaks, then people would rationally form priors about it. Second, and most importantly,
} 
to $\$ p$ per viewer reached for placing an advertisement during a program in each period.

On the other side of the market, there is a continuum of $N$ potential viewers who are uniformly distributed on the unit line with respect to their ideal program types. To each possible program type on the unit line, there corresponds a viewer for whom that program is the ideal one. Individuals have the same program preferences during both periods. An individual derives $v$ units of utility from watching her ideal program that carries $A$ nonprogram breaks. ${ }^{3}$ Formally, a viewer who is located at a distance of $d$ units from a program obtains a net viewing benefit $v-d$. Not watching TV yields zero benefits. ${ }^{4}$ The parameter $\lambda$ will be used to represent the location of an individual, and a particular individual will be referred to as "she" when it is convenient.

The locations of the first programs is assumed to be common knowledge. Although people know that each station offers two consecutive program, they do not know where on the unit line the second programs are located. Denoting the location of second program of station $Y$ with $y$ and that of $Z$ with $z$, I assume that viewers' priors are given by $y, z \in\left\{0, \frac{1}{2}, 1\right\}$. From their perspective, each of these three locations is equally likely to be the actual location of each program. The stations know the location of their own as well as their rival's program, and people know that the stations have this information. They may devote one of the nonprogram breaks in the first period to a tune-in. ${ }^{5}$ A tune-in may only include information about the actual location of the upcoming program at the same station. I assume that the TV stations cannot lie, i.e. they are legally bound to advertise a preview of the actual upcoming program in the tune-in. The objective of the TV stations is to maximize total advertisement revenues (for simplicity, it is assumed that there is no discounting).

the number of non-program breaks in the first period would provide information about the second-period programs.

${ }^{3}$ The base utility, $v$, also captures the effects of the disutility associated with interruptions during a program. Specifically, the effect of an increase (a decrease) in the nuisance cost of a non-program break on a viewer's utility can be captured by lowering (raising) the base utility.

${ }^{4} \mathrm{We}$ can as well include a constant, $t$, in front of $d$ that measures the disutility associated with one unit of distance from the ideal program type. However, since the value of not watching TV is zero, we can easily express the utility as $r-d$, where $r=\frac{v}{t}$.

${ }^{5}$ They would never air more than one tune-in because tune-ins are assumed to be fully informative, and viewers do not switch stations in the first period. 
Viewers have the option of switching to the other station or simply turning the TV off after sampling a few minutes of a program. I assume that the amount of time required for learning the true location of a program is fixed and same for both programs and for all individuals. This sampling process entails a cost of $c$, and is referred to as the "sampling cost". A viewer incurs one unit of the sampling cost if she samples the programs at both stations and ends up watching the one that yields a higher utility. If an individual decides the turn her TV off after sampling one of the programs, then her net utility is $-c$. If she does so after sampling both programs, then her net utility is $-2 c$. Since the locations of the first programs are known beforehand, viewers do not engage in sampling in the first period. However, sampling one or both of the stations may be optimal in the second period. An individual's objective is to make a decision at each time that maximizes her total utility.

I maintain the following three assumptions throughout the analysis.

Assumption $1 \frac{1}{4}+c<v<\frac{1}{2}-c$, where $c>0$.

Assumption $2 \frac{1}{4}+\frac{1}{2 A}<v<\frac{1}{2}-\frac{1}{A}$, where $A>0$.

Assumption 3 The first programs at stations $Y$ and $Z$ are located at $\frac{1}{4}$ and $\frac{3}{4}$, respectively, and this is common knowledge.

The first and the second assumptions are made in order to rule out unreasonable equilibria. This shall be more clear as the analysis proceeds. Note that it imposes an upper bound on the value of the sampling cost, and a lower bound on the number of non-program breaks. To be more specific, it is implied that $0<c<\frac{1}{8}$ and $A>6$. The third assumption is made in order to simplify the analysis. Combined with the first assumption, it implies that viewers on the lower half of the unit line watch station $Y$ and the ones on the upper half watch station $Z$.

The timing of the moves is as follows. First, people make their first period viewing decisions. Then the first program starts, and the TV stations make their tune-in decisions during the first program. Having watched the first program, people update their beliefs 
about the second programs depending on whether or not they were exposed to a tune-in. The second programs start and people make their optimal sampling decisions. After each individual completes sampling one or both (or none) of the stations, they make their final second period viewing choices and the payoffs are realized.

\subsection{Equilibrium}

The equilibrium concept used is perfect Bayesian equilibrium (PBE). That is, the TV stations make optimal tune-in decisions taking the location of their rival's program and the rationality of people into account, and people make optimal sampling and viewing decisions after observing the tune-in decision of the station they have watched. In particular, people's inferences (or posterior beliefs) after the first period about the locations of the second programs must be correct, and the TV stations should not have any incentive to deviate.

As discussed in the Introduction, the TV stations may choose to behave strategically due to their knowledge of the rival station's program. However, regardless of the location of the rival's program, a station clearly never airs a tune-in for a program that none of its current viewers would like to watch. This case arises for station $Y$ when $y=1$, and for station $Z$ when $z=0$. Given that a station cannot communicate any information with the viewers of the other station, it does not pay off for either station to air a tune-in for such programs. Let $\beta_{i}(y, z)$ be a binary variable that assumes a value of 1 if station $i$ airs a tune-in when the two programs are located at $(y, z)$, and 0 otherwise. So, if station $Y$ airs a tune-in for $y=0$ when $z=0$, then we have $\beta_{Y}(0,0)=1$. The following lemma is immediate.

Lemma $1 \beta_{Y}(1, z)=0$ for all $z$, and $\beta_{Z}(y, 0)=0$ for all $y$.

Next, consider a situation in which neither of the stations air a tune-in for their upcoming programs regardless of their locations. Suppose that these strategies constitute a PBE. In such a "no tune-in" equilibrium, people's priors would be unchanged. This means that all viewers are indifferent between sampling either station. So, if sampling occurs, a random half of viewers initially sample $Y$ and the remaining ones initially sample $Z$. Suppose a $\lambda$-type 
viewer chooses to sample one of the programs. If $\lambda$ is such that $0 \leq \lambda \leq \frac{1}{2}-v-c$, then this viewer knows that she would only watch a program located at 0 . If the program that she first samples is not at 0 , should she also sample the program at the other station? Unless the other program happens to be located at 0, she would turn her TV off, and her net utility would be $-2 c$ since she would have sampled both programs and ended up taking the outside option. So, the expected utility of sampling the other station is $\frac{1}{3}(v-c-\lambda)+\frac{2}{3}(-2 c)$. On the other hand, if she switches off without sampling the other station, she would enjoy a utility of $-c$. Hence, she should engage in a second sampling if $\frac{1}{3}(v-c-\lambda)+\frac{2}{3}(-2 c) \geq-c$, or equivalently if $v-\lambda \geq 2 c$. The left-hand side is decreasing in $\lambda$, so if this inequality is satisfied at $\lambda=\frac{1}{2}-v-c$, it has to be true for all $\lambda \leq \frac{1}{2}-v-c$. Evaluating at $\lambda=\frac{1}{2}-v-c$, we get $2 v-c \geq \frac{1}{2}$ which is always true by Assumption (1).

We also need to check if engaging in sampling is optimal at all for this person. Expected utility of doing so is $\frac{1}{3}(v-\lambda)+\frac{2}{3}\left[\frac{1}{3}(v-c-\lambda)+\frac{2}{3}(-2 c)\right]$, where the second term is due to the fact that it is also optimal to sample the other station when the first program sampled is not at 0 . If this is nonnegative, then it is optimal to engage in sampling for viewers with locations $\lambda \leq \frac{1}{2}-v-c$. Rearranging, expected utility becomes $\frac{1}{3}[v-\lambda-2 c]$, which is the same condition as in the previous paragraph, and therefore is nonnegative.

Now, take a viewer with location $\lambda \in\left[\frac{1}{2}-v-c, \frac{1}{4}\right]$ and suppose that this viewer samples station $Y$. She stays at $Y$ if $y$ is located at 0 . If it turns out that $y=\frac{1}{2}$, she may also want to check out station $Z$ in the hope of finding out $z=0$. But there is also the chance that $z$ is $\frac{1}{2}$ or 1 . If $z=1$, she would switch back to station $Y$. If, on the other hand, $z=\frac{1}{2}$, she would be indifferent between the two stations. So, the expected utility of switching to station $Z$ when $y=\frac{1}{2}$ is $\frac{1}{3}(v-c-\lambda)+\frac{2}{3}\left(v-c-\frac{1}{2}+\lambda\right)$. If this expression is greater than the utility of staying at $Y, v-\left(\frac{1}{2}-\lambda\right)$, she should switch to and sample the program at station $Z$. This is satisfied when $\lambda<\frac{1}{4}-\frac{3 c}{2}$. So, when $y=\frac{1}{2}$, it is optimal to also sample $Z$ for the viewers with locations $\frac{1}{2}-v-c \leq \lambda<\frac{1}{4}-\frac{3 c}{2}$. Finally, suppose it turns out that $y=1$. The expected utility of switching to $Z$ is $\frac{1}{3}(v-c-\lambda)+\frac{1}{3}\left(v-c-\frac{1}{2}+\lambda\right)+\frac{1}{3}(-2 c)$ which equals $\frac{1}{3}\left(2 v-4 c-\frac{1}{2}\right)$. This is greater than $-c$ when $2 v-c>\frac{1}{2}$, which is again true 
by Assumption (1).

The analysis above equally applies to other possibilities as well. So, as a general rule, stopping sampling is optimal when the location of the program first sampled is at most at a distance $\frac{1}{4}+\frac{3 c}{2}$ from a viewer's own location. We can now express the audience shares of stations $Y$ and $Z$ for all possible values of $(y, z)$ under the assumption that there are no tune-ins. Suppose, for instance, that $(y, z)=\left(0, \frac{1}{2}\right)$. A random half of the viewers sample station $Y$ first. Among these viewers, those with $\lambda \leq \frac{1}{4}+\frac{3 c}{2}$ stay at $Y$ while the rest switch to $Z$. Since $z=\frac{1}{2}$, those with $\lambda>\frac{1}{2}+v+c$ turn their TVs off. From among the other half who chose to sample $Z$ first, the ones with $\lambda \in\left[\frac{1}{4}-\frac{3 c}{2}, \frac{3}{4}+\frac{3 c}{2}\right]$ stay at $Z$ while the others switch to $Y$. Those with $\frac{1}{4}-\frac{3 c}{2}<\lambda$ stay at $Y$. The same is not true for $\lambda>\frac{3}{4}+\frac{3 c}{2}$. The program $y=0$ is not favorable for them, so those with locations $\lambda \in\left[\frac{3}{4}+\frac{3 c}{2}, \frac{1}{2}+v+c\right]$ switch back to station $Z$ while the rest switch off. Arguing along similar lines, we get the following audience shares:

\begin{tabular}{|l|c|c|c|}
\hline & $\mathbf{z}=\mathbf{0}$ & $\mathbf{z}=\frac{1}{2}$ & $\mathbf{z}=\mathbf{1}$ \\
\hline $\mathbf{y}=\mathbf{0}$ & $\frac{v+c}{2}$ & $\frac{1}{4}$ & $v+c$ \\
\hline $\mathbf{y}=\frac{1}{2}$ & $v+c+\frac{1}{4}$ & $v+c$ & $v+c+\frac{1}{4}$ \\
\hline $\mathbf{y}=\mathbf{1}$ & $v+c$ & $\frac{1}{4}$ & $\frac{v+c}{2}$ \\
\hline
\end{tabular}

Table 1a. Audience share of station $Y$ in the "no tune-in" PBE

\begin{tabular}{|c|c|c|c|}
\hline & $\mathbf{z}=\mathbf{0}$ & $\mathbf{z}=\frac{1}{2}$ & $\mathbf{z}=\mathbf{1}$ \\
\hline $\mathbf{y}=\mathbf{0}$ & $\frac{v+c}{2}$ & $v+c+\frac{1}{4}$ & $v+c$ \\
\hline $\mathbf{y}=\frac{1}{2}$ & $\frac{1}{4}$ & $v+c$ & $\frac{1}{4}$ \\
\hline $\mathbf{y}=\mathbf{1}$ & $v+c$ & $v+c+\frac{1}{4}$ & $\frac{v+c}{2}$ \\
\hline
\end{tabular}

Table 1b. Audience share of station $Z$ in the "no tune-in" PBE

Now, suppose $Y$ aired a tune-in when $(y, z)=(0,0)$. For the indifferent viewer from the first-period audience of station $Y$, the expected utility of switching to $Z$ is $\frac{1}{3}(v-\lambda)+$ $\frac{1}{3}\left(v-\frac{1}{2}+\lambda\right)+\frac{1}{3}(v-c-\lambda)$, where the first term is the utility she would enjoy at $Z$ when $z=0$, the second term is the utility she would enjoy at $Z$ when $z=\frac{1}{2}$, and the third term is the utility she would enjoy at $Y$ when $z=1$. This expression equals the utility of staying at $Y, v-\lambda$, for the viewer located at $\frac{1}{4}+\frac{c}{2}$, so the viewers with $\lambda \leq \frac{1}{4}+\frac{c}{2}$ do not switch to 
$Z$. Since this is a unilateral deviation, the behavior of the first-period viewers of station $Z$ remains the same. The ones who switch to $Z$ do not come back to $Y$ since they would incur the sampling cost. So, station $Y$ would gain an extra audience of $\left(\frac{1}{4}+\frac{c}{2}\right)-\left(\frac{v+c}{2}\right)=\frac{1-2 v}{4}$ by airing a tune-in, and thus its second-period advertising revenue would go up by $A N p\left(\frac{1-2 v}{4}\right)$. The cost of airing a tune-in is the revenue forgone in the first period from a single commercial, which is $N p\left(\frac{1}{2}\right)$. Thus, it is profitable to deviate as long as $\frac{1}{2}-v \geq \frac{1}{A}$.

It will prove useful to analyze in more detail the incentive for airing a tune-in when either station starts from a no tune-in situation. I will henceforth call this situation the "no tune-in" regime. Suppose the program locations are $(y, z)=\left(\frac{1}{2}, \frac{1}{2}\right)$. If neither station airs a tune-in, they each receive an expected audience size of $v+c$ in the second period. From the previous analysis, if station $Y$ airs a tune-in, viewers with locations $\lambda \in\left[\frac{1}{4}-\frac{c}{2}, \frac{1}{2}\right]$ continue to stay with $Y$ while the others, $\lambda<\frac{1}{4}-\frac{c}{2}$, switch to $Z$. The ones who switch to $Z$ will stay there once they discover that $z=\frac{1}{2}$, since switching back to $Y$ means missing the first few minutes of the program at $Y$. When this is a unilateral deviation by $Y$, behavior of the first-period viewers of $Z$ does not change. A random half of them still switch to $Y$ right after the first period ends. Among these viewers, the ones with locations $\lambda \in\left[\frac{1}{2}, \frac{3}{4}+\frac{3 c}{2}\right]$ stay at $Y$. The others go back to $Z$ to check out the program there. Once they discover that $z=\frac{1}{2}$, those with locations at most $v+c$ apart from $\frac{1}{2}$ choose one of the stations at random as their final destination. Similarly, among those who stayed at $Z$ at first, $\lambda \in\left[\frac{3}{4}+\frac{3 c}{2}, 1\right]$ also sample $Y$ and a random half of $\lambda \in\left[\frac{3}{4}+\frac{3 c}{2}, \frac{1}{2}+v+c\right]$ stay at $Y$. Hence, station $Y$ collects a total audience size of $\left(\frac{1}{4}+\frac{c}{2}\right)+\frac{1}{2}(v+c)$ as opposed to $v+c$. Since this is a symmetric game, station $Z$ will have the same incentives. When both stations air a tune-in, they each get an audience of $v+c$, the same as before but with different composition. Thus, we get the following audience sizes when $(y, z)=\left(\frac{1}{2}, \frac{1}{2}\right)$,

\begin{tabular}{|c|c|c|}
\hline & $\boldsymbol{\beta}_{Z}=\mathbf{0}$ & $\boldsymbol{\beta}_{Z}=\mathbf{1}$ \\
\hline $\boldsymbol{\beta}_{Y}=\mathbf{0}$ & $v+c, v+c$ & $\frac{3 v}{2}-\frac{1}{4}+c, \frac{v}{2}+\frac{1}{4}+c$ \\
\hline $\boldsymbol{\beta}_{Y}=\mathbf{1}$ & $\frac{v}{2}+\frac{1}{4}+c, \frac{3 v}{2}-\frac{1}{4}+c$ & $v+c, v+c$ \\
\hline
\end{tabular}

Table 2. Audience sizes of $(Y, Z)$ in a "no tune-in" regime when $(y, z)=\left(\frac{1}{2}, \frac{1}{2}\right)$ 
The unique Nash equilibrium in this game is $\left(\beta_{Y}, \beta_{Z}\right)=(1,1)$ provided that $\frac{1}{2}-v \geq \frac{1}{A}$. This is true by Assumption (1) and thus the stations face a Prisoners' Dilemma situation. That is why a "no tune-in" regime cannot be maintained in an equilibrium.

Lemma 2 By Assumptions (1) and (2), it must be true that $\beta_{Y}(0,0)=\beta_{Y}\left(\frac{1}{2}, \frac{1}{2}\right)=$ $\beta_{Z}\left(\frac{1}{2}, \frac{1}{2}\right)=\beta_{Z}(1,1)=1$ is satisfied in a symmetric PBE.

Note that when $(y, z)=\left(0, \frac{1}{2}\right)$ or $\left(\frac{1}{2}, 0\right)$, deviating from a "no tune-in" regime is profitable for station $Y$ if $A\left[\left(\frac{1}{4}+\frac{c}{2}\right)-\frac{1}{4}\right] \geq \frac{1}{2}$, or equivalently if $A c \geq 1$. Although this condition is different from what was shown to be necessary for deviation when $(y, z) \in\left\{(0,0),\left(\frac{1}{2}, \frac{1}{2}\right)\right\}$, it is in general more restrictive compared to $\frac{1}{2}-v \geq \frac{1}{A}$. Together with Assumption (1), $A c \geq 1$ implies $\frac{1}{2}-v \geq \frac{1}{A}$. However, $\frac{1}{2}-v \geq \frac{1}{A}$ does not necessarily imply $A c \geq 1$. An important thing to note is that viewers' optimal sampling behavior depends on their inferences from the observed tune-in decisions of the TV stations. As will be argued shortly, a strategy in which station $Y$ airs a tune-in only when $(y, z) \in\left\{(0,0),\left(\frac{1}{2}, \frac{1}{2}\right)\right\}$ cannot actually be part of a PBE. So, it is not nesessary to assume that $A c \geq 1$ for this outcome to arise.

To see this, consider an equilibrium in which station $Y$ airs a tune-in only when $(y, z) \in$ $\left\{(0,0),\left(\frac{1}{2}, \frac{1}{2}\right)\right\}$, and station $Z$ does so only when $(y, z) \in\left\{\left(\frac{1}{2}, \frac{1}{2}\right),(1,1)\right\}$. For these strategies to constitute a PBE, viewers' inferences from observed tune-in decisions must be correct. Therefore, when station $Y$ advertises $y=0$, the first-period viewers of $Y$ infer that $z=0$ as well. This means that each station ends up with an audience size of $\frac{v}{2}$ as each viewer will watch the first station they choose to sample. Both stations are actually worse off compared to the "no tune-in" regime. However, it is in fact optimal for station $Y$ to switch back to the "no tune-in" regime if $Z$ is going to air a tune when $(y, z) \in\left\{\left(\frac{1}{2}, \frac{1}{2}\right),(1,1)\right\}$. If a $\lambda$-type viewer who is initially indifferent between the two stations continues to stay at $Y$ after not seeing a tune-in, she will infer (incorrectly) that $z$ is either $\frac{1}{2}$ or 1 upon seeing that $y=0$. If $\lambda>\frac{1}{4}+c$, it is worth checking out station $Z$, too. But when she discovers that $z$ is also 0 , she will one more time be indifferent between the two stations provided that $\lambda \leq v+c$. Similarly, if she starts at $Z$ and sees that $z=0$, she will infer that $y$ is either $\frac{1}{2}$ or 1 , and will 
switch to $Y$ if $\lambda>\frac{1}{4}+c$. The second-period audience size of station $Y$ is thus $\frac{v+c}{2}$ as opposed to $\frac{v}{2}$, which means that station $Y$ has an incentive to not air a tune-in when $(y, z)=(0,0)$. But viewers anticipate this correctly and it was previously shown that this cannot be an equilibrium either.

One way to get around this problem is airing a tune-in more often. That is, when a TV station airs a tune-in for a particular program location, its viewers should not be able to infer the exact location of the other program. For station $Y$, the strategy of airing a tune-in for $y=0$ only when $z=0,1$ (similarly, the strategy $\beta_{Y}\left(\frac{1}{2}, z\right)=1$ only when $z=\frac{1}{2}, 1$ ) cannot happen in equilibrium. This is because station $Y$ would also air a tune-in when $(y, z)=\left(0, \frac{1}{2}\right)$ so as to (incorrectly) signal to its viewers that $z$ is either 0 or 1 . So, no firstperiod viewer of $Y$ would switch to $Z$, and thus $Y$ would get an audience size of $v$. If instead $Y$ did not air a tune-in - as would be anticipated by viewers in a PBE - all of its current viewers would switch to station $Z$ and would infer that $y$ is either 0 or 1 upon seeing that $z=\frac{1}{2}$. In this case, those viewers with $\lambda<\frac{1}{4}-c$ would switch back to station $Y$ and stay there upon discovering $y=0$. So, airing a tune-in is profitable when $v-\left(\frac{1}{4}-c\right) \geq \frac{1}{2 A}$, which is true by Assumption (2). Note that this condition is satisfied even when the sampling cost is infinitesimally small.

This leaves us with two possible strategies. For station $Y$, these strategies are (i) air a tune-in unless $y$ is 1 , (ii) air a tune-in unless $y$ or $z$ is 1 . Similarly, airing a tune-in unless $z=0$ and unless $z$ or $y=0$ are the only two possible strategies for station $Z$. In what follows, I will refer to a strategy in which a station's tune-in decision does not depend on the program of the other station as a non-strategic behavior, and to an equilibrium that involves non-strategic behavior as a non-strategic equilibrium. Similarly, a tune-in strategy that depends on the program of the other station will be referred as a strategic behavior, and the corresponding equilibrium as a strategic equilibrium.

Suppose each station behaves strategically. How would viewers behave if this were a PBE? Since the two stations are identical in every aspect except for the locations of the first programs, the viewing behavior of people in the second period will be symmetric with 
respect to which station they watched in the first period. Therefore, I will only find the optimal sampling and final viewing decisions of the viewers who chose to watch $Y$ in the first period. There are three cases; $Y$ airs a tune-in for $y=0, Y$ airs a tune-in for $y=\frac{1}{2}$, and $Y$ does not air a tune-in.

Case (1): $Y$ airs a tune-in for $y=0$.

In this case, the viewers of $Y$ infer that $z \in\left\{0, \frac{1}{2}\right\}$. Those with locations closer to $\frac{1}{2}$ will have a tendency to switch to $Z$. Whatever the location of $z$ turns out, none of these people would come back to $Y$. So, the solution is simple; $\lambda \leq \frac{1}{4}$ stay with $Y$, the others switch to $Z$. Those who switch to $Z$ will have the sampling cost sunk, and therefore $\frac{1}{4}<\lambda \leq v+c$ will stay with $Z$ when $z=0$. The others just switch off in this case. If $z$ turns out $\frac{1}{2}$, then all of them stay with $Z$.

Case (2): $Y$ airs a tune-in for $y=\frac{1}{2}$.

In this case, the viewers of $Y$ infer that $z \in\left\{0, \frac{1}{2}\right\}$. Those with locations closer to 0 will have a tendency to switch to $Z$. Similar with case (1), $\lambda \geq \frac{1}{4}$ stay with $Y$, the others switch to $Z$. Those who switch to $Z$ will have the sampling cost sunk, and therefore $\frac{1}{2}-(v+c)<\lambda \leq \frac{1}{2}$ will stay with $Z$ when $z=\frac{1}{2}$. If $z$ turns out 0 , then all of them stay with $Z$.

Case (3): $Y$ does not air a tune-in.

The inference of viewers in this case is that $Y$ did not air a tune-in because either $y=1$ or $z=1$ (or both). There are five possibilities:

$$
(y, z) \in\left\{(0,1),\left(\frac{1}{2}, 1\right),(1,0),\left(1, \frac{1}{2}\right),(1,1)\right\}
$$

So the posterior probability that $y=0$ is same with the probability that $z=0$, which is $\frac{1}{5}$. Similarly, $\operatorname{Pr}\left(y=\frac{1}{2}\right)=\operatorname{Pr}\left(z=\frac{1}{2}\right)=\frac{1}{5}$, and $\operatorname{Pr}(y=1)=\operatorname{Pr}(z=1)=\frac{3}{5}$. This means that viewers are indifferent between the two stations, and a random half will choose $Z$ first. For those who stayed with $Y$, the actual location of $y$ will determine their further behavior.

If $y=0$, they infer that $z=1$. So viewers with locations less than $v+c$ stay with $Y$, and the rest switch off. Note that for $v<\lambda \leq v+c$ switching off yields a disutility of $c$, so it is better to stay tuned. 
If $y=\frac{1}{2}$, they infer that $z=1$. So viewers with locations $\frac{1}{2}-(v+c) \leq \lambda \leq \frac{1}{2}$ stay with $Y$, and the rest switch off.

If $y=1$, they infer that $z \in\left\{0, \frac{1}{2}, 1\right\}$, each with equal probability. Viewers with locations $0 \leq \lambda<\frac{1}{2}-(v+c)$ would stay with $Z$ when $z=0$, should they sample it. So, the expected utility of sampling $Z, E\left[U_{Z}^{\lambda}\right]$, given that station $Y$ does not air a tune-in, $\beta_{Y}^{-1}(0)=(1, z)$, is,

$$
E\left[U_{Z}^{\lambda} \mid \beta_{Y}^{-1}(0)=(1, z)\right]=\frac{1}{3}(v-c-\lambda)-\frac{2}{3}(2 c)
$$

Note that the highest utility a viewer may get in this case is $v-c$, since she started sampling with station $Y$ and incurred the sampling cost. Viewers would stay tuned if the expected utility of sampling $Z$ is not less than $-c$. Otherwise they turn their TVs off right after the first program ends. For $\lambda=\frac{1}{2}-(v+c)$, the expected utility of sampling is $\frac{1}{3}\left(2 v-\frac{1}{2}\right)-\frac{2}{3}(2 c)$. This is at least as great as $-c$ if $2 v-\frac{1}{2} \geq c$, which is true by Assumption (1). Since $E\left[U_{Z}^{\lambda} \mid \beta_{Y}^{-1}(0)=(1, z)\right]$ is decreasing in $\lambda$, all of these viewers would choose to sample $Z$. Viewers with locations $\frac{1}{2}-(v+c)<\lambda \leq \frac{1}{4}$ would stay with $Z$ unless $z=1$. So, their expected utility is,

$E\left[U_{Z}^{\lambda} \mid \beta_{Y}^{-1}(0)=(1, z)\right]=\frac{1}{3}\left[(v-c-\lambda)+\left(v-c-\left(\frac{1}{2}-\lambda\right)\right)-(2 c)\right]=\frac{1}{3}\left(2 v-4 c-\frac{1}{2}\right)$

This expression is greater than or equal to $-c$ when $2 v-\frac{1}{2} \geq c$, which is the same condition as before. Hence, it is satisfied for all $\lambda \in\left[\frac{1}{4}, \frac{1}{2}\right]$. The choices of viewers with locations on $\left[\frac{1}{4}, \frac{1}{2}\right]$ are just symmetric with those on $\left[0, \frac{1}{4}\right]$, so they all sample $Z$ as well. If the program turns out to be located at 0 or $\frac{1}{2}$, station $Z$ gets an audience size of $N(v+c)$. If $z=1$, everyone switches off.

For those of $0 \leq \lambda \leq \frac{1}{2}$ who switched to $Z$ initially, the subsequent choices are similar. Now, I need to check if sampling one of the stations is desirable at all, conditional on not seeing a tune-in. For $0 \leq \lambda<\frac{1}{2}-(v+c)$, the expected utility of starting sampling with station $Y$ is,

$$
E\left[U_{Z}^{\lambda} \mid \beta_{Y}=0\right]=\frac{1}{5}(v-\lambda)+\frac{1}{5}(-c)+\frac{3}{5} E\left[U_{Z}^{\lambda} \mid \beta_{Y}^{-1}(0)=(1, z)\right]
$$


Similarly, for $\frac{1}{2}-(v+c) \leq \lambda<\frac{1}{4}$, it is,

$$
E\left[U_{Z}^{\lambda} \mid \beta_{Y}=0\right]=\frac{1}{5}(v-\lambda)+\frac{1}{5}\left(v-\frac{1}{2}+\lambda\right)+\frac{3}{5} E\left[U_{Z}^{\lambda} \mid \beta_{Y}^{-1}(0)=(1, z)\right]
$$

We need this value to be nonnegative for a viewer to sample $Y$. For $0 \leq \lambda<\frac{1}{2}-(v+c)$, $E\left[U_{Z}^{\lambda} \mid \beta_{Y}=0\right]=\frac{2}{5}(v-c-\lambda)-\frac{2}{5}(2 c)$. This is negative if $\lambda$ is greater than $3 c-v$. If $\frac{1}{2}-(v+c)$ is less than (or equal to) $3 c-v$, then all of these people engage in sampling. $\frac{1}{2}-(v+c) \leq 3 c-v$ if $c \geq \frac{1}{8}$. By Assumption (1), we must have $\frac{1}{4}+c<\frac{1}{2}-c$, which implies $c<\frac{1}{8}$. By monotonicity of $E\left[U_{Z}^{\lambda} \mid \beta_{Y}=0\right]$ (increasing up to $\lambda=\frac{1}{4}$, and decreasing thereafter), we can conclude that sampling is desirable conditional on $\beta_{Y}=0$.

We are now ready to calculate the audience share of a station. The table below gives the total fraction of the population choosing station $Y$ to watch (after sampling, if any) in the second period for all possible program locations.

\begin{tabular}{|l|c|c|c|}
\hline & $\mathbf{z}=\mathbf{0}$ & $\mathbf{z}=\frac{1}{2}$ & $\mathbf{z}=\mathbf{1}$ \\
\hline $\mathbf{y}=\mathbf{0}$ & $\frac{1}{4}$ & $\frac{1}{4}$ & $v+c$ \\
\hline $\mathbf{y}=\frac{1}{2}$ & $\frac{1}{4}+(v+c)$ & $v+c$ & $(v+c)+\frac{1}{4}$ \\
\hline $\mathbf{y}=\mathbf{1}$ & $v+c$ & $\frac{1}{4}$ & $(v+c)-\frac{1}{4}$ \\
\hline
\end{tabular}

Table 3. Audience share of $Y$ in the strategic PBE

Does station $Y$ have any incentive to deviate? Suppose $(y, z)=(0,0)$. If station $Y$ deviates and does not air a tune-in, then a random half of its viewers stay with it while the other half switch. Those who stayed would think that $z=1$ upon seeing that $y=0$, and the ones with locations less than $v+c$ would continue staying. Those who initially switched to $Z$ would think that $y=1$ upon seeing $z=0$, and therefore none of them would switch back to $Y$. So, station $Y$ would end up with an audience share of $\frac{v+c}{2}$. It is profitable to deviate if

$$
A\left(\frac{1}{4}-\frac{v+c}{2}\right)<\frac{1}{2}
$$

where the left hand side is the marginal per-viewer revenue of a tune-in and the right hand side is the per-viewer cost of a tune-in. So, $Y$ would not deviate if $v+c+\frac{1}{A} \leq \frac{1}{2}$. The same is true for $(y, z)=\left(0, \frac{1}{2}\right),\left(\frac{1}{2}, 0\right)$ and $\left(\frac{1}{2}, \frac{1}{2}\right)$. Note that deviation is not profitable when 
$y=1$ since station $Y$ can only communicate with its own viewers, and none of them would watch a program located at 1 . It remains to analyze if it is profitable for $Y$ to deviate when $(y, z)=(0,1)$ or $\left(\frac{1}{2}, 1\right)$. In both cases, station $Y$ is already getting the highest possible audience share from its first period without a tune-in. So, airing a tune-in cannot increase $Y$ 's audience size. Therefore, deviation is not profitable in these two cases, either.

Proposition 1 The following constitutes a symmetric PBE if $v+c+\frac{1}{A} \leq \frac{1}{2}: Y$ airs a tune-in unless $y$ or $z$ is $1, Z$ airs a tune-in unless $y$ or $z$ is 0 .

When $v+c+\frac{1}{A}>\frac{1}{2}$, people have no reason to expect the strategies in Proposition (1) to be played by the TV stations. This condition is satisfied when $v+c$ is large and/or the number of non-program breaks is small. Intuitively, a larger value of $v$ is associated with a higher audience size since more viewers end up watching TV. A higher sampling cost means that if sampling occurs in the absence of a tune-in, a higher fraction of those who sample stay tuned. When the number of non-program breaks is small, the marginal benefit of promoting the upcoming program is lower. So, in all three cases, the incentive for passing up on airing a tune-in is higher.

From viewers' point of view, the ex-ante expected value of station $Y$ 's per-viewer profits is the weighted average of the profits in each of the possible nine cases. Per-viewer revenue in the first period is $\frac{p A}{2}$ in each case. Per-viewer revenue in the second period is the average of the audience shares given in the table for all of the nine cases, multiplied with $p A$. Since $Y$ is expected to air a tune-in in four of the nine cases, its expected per-viewer costs are $\frac{4 p}{9}$ times the audience share in the first period (which is $\frac{1}{2}$ ). So, the ex-ante expected per-viewer profits of station $Y$ can be expressed as (the superscript $S$ stands for strategic),

$$
E\left[\Pi_{j}^{S}\right]=\left[\frac{A}{2}+\frac{(6(v+c)+1) A}{9}-\frac{2}{9}\right] p, \quad j=Y, Z
$$

What happens when $v+c+\frac{1}{A}>\frac{1}{2}$ ? Based on the analysis so far, one possibility is when each station airs an additional tune-in relative to the PBE in Proposition (1). For station $Y$, this is when $\beta_{Y}(0, z)=1$ for all $z$ and $\beta_{Y}\left(\frac{1}{2}, z\right)=1$ unless $z=1$, or $\beta_{Y}\left(\frac{1}{2}, z\right)=1$ for all 
$z$ and $\beta_{Y}(0, z)=1$ unless $z=1$ (symmetric for $Z$ ). These are summarized in the following table (for station $Y$ only):

Strategy 1

\begin{tabular}{|c|c|c|c|}
\hline & $\mathbf{z}=\mathbf{0}$ & $\mathbf{z}=\frac{1}{2}$ & $\mathbf{z}=\mathbf{1}$ \\
\hline $\mathbf{y}=\mathbf{0}$ & 1 & 1 & 1 \\
\hline $\mathbf{y}=\frac{1}{2}$ & 1 & 1 & 0 \\
\hline $\mathbf{y}=\mathbf{1}$ & 0 & 0 & 0 \\
\hline
\end{tabular}

Strategy 2

\begin{tabular}{|c|c|c|c|}
\hline & $\mathbf{z}=\mathbf{0}$ & $\mathbf{z}=\frac{1}{2}$ & $\mathbf{z}=\mathbf{1}$ \\
\hline $\mathbf{y}=\mathbf{0}$ & 1 & 1 & 0 \\
\hline $\mathbf{y}=\frac{1}{2}$ & 1 & 1 & 1 \\
\hline $\mathbf{y}=\mathbf{1}$ & 0 & 0 & 0 \\
\hline
\end{tabular}

Table 4. The value of $\beta_{Y}(y, z)$ in two alternative regimes

When $Y$ does not air a tune-in, all of its viewers will switch to $Z$ since it is highly likely that $y=1$. If $z$ turns out 0 or $\frac{1}{2}$, then they are certain that $y=1$, and none of them come back to $Y$. When it turns out that $z=1$, however, viewers will get confused. Station $Y$ might have played the first or the second strategy. If it played the first strategy, $y$ could be $\frac{1}{2}$ or 1 with equal chances. If it played the second one, on the other hand, $y$ is 0 or 1 . Without any further information, viewers just assume that two strategies are equally likely to be played, and therefore their inference will be $\operatorname{Pr}(y=0)=\operatorname{Pr}\left(y=\frac{1}{2}\right)=\frac{1}{4}, \operatorname{Pr}(y=1)=\frac{1}{2}$. But sampling $Y$ would be optimal for all $\lambda \in\left[0, \frac{1}{2}\right]$ with these posteriors. This means that station $Y$ could have done better by deviating, and reverting back to the strategy in Proposition (1). Since viewers anticipate this beforehand, we cannot have either of these strategies being played in a symmetric PBE.

A second, and the only other, possibility when $v+c+\frac{1}{A}>\frac{1}{2}$ is the non-strategic equilibrium in which the TV stations air a tune-in unless their program is located at the other end of the unit line. In this case, the priors of viewers who watch $Y$ in the first period about $z$ are unchanged regardless of the tune-in decision of $Y$.

Case (1): $Y$ airs a tune-in for $y=0$.

Since there is also the chance that $z=1$, only the viewers with $\lambda>\frac{1}{4}+\frac{c}{2}$ sample $Z$. If $z$ turns out to be located at 1 , the ones with $\lambda \leq v$ come back to $Y$. If $z=0$ or $\frac{1}{2}$, none of them come back. 
Case (2): $Y$ airs a tune-in for $y=\frac{1}{2}$.

This case is symmetric with Case (1).

Case (3): $Y$ does not air a tune-in.

In this case, it is inferred that $y=1$, and therefore none of the current viewers of $Y$ will watch it.

Calculating the audience share of station $Y$ for all possible program locations gives,

\begin{tabular}{|c|c|c|c|}
\hline & $\mathbf{z}=\mathbf{0}$ & $\mathbf{z}=\frac{1}{2}$ & $\mathbf{z}=\mathbf{1}$ \\
\hline $\mathbf{y}=\mathbf{0}$ & $\frac{1}{4}+\frac{c}{2}$ & $\frac{1}{4}+\frac{c}{2}$ & $v$ \\
\hline $\mathbf{y}=\frac{1}{2}$ & $\frac{1}{4}+\frac{c}{2}+(v+c)$ & $v+c$ & $v+\frac{1}{4}-\frac{c}{2}$ \\
\hline $\mathbf{y}=\mathbf{1}$ & $v+c$ & $\frac{1}{4}-\frac{c}{2}$ & $v+c-\left(\frac{1}{4}+\frac{c}{2}\right)$ \\
\hline
\end{tabular}

Table 5. Audience share of station $Y$ in the non-strategic PBE

Note that deviation is not possible in this case, since none of $Y$ 's current viewers would keep watching or would come back later when $\beta_{Y}=0$. So, the unique symmetric PBE when $v+c+\frac{1}{A}>\frac{1}{2}$ is the one in which the two stations play non-strategically.

Proposition 2 When $v+c+\frac{1}{A}>\frac{1}{2}$, the unique symmetric PBE is the one in which $Y$ airs a tune-in unless $y=1$, and $Z$ airs a tune-in unless $z=0$.

Arguing along the same lines as before, the ex-ante expected per-viewer profits of a station can be expressed as (the superscript $N S$ stands for non-strategic),

$$
E\left[\Pi_{j}^{N S}\right]=\left[\frac{A}{2}+\frac{(6 v+4 c+1) A}{9}-\frac{1}{3}\right] p, \quad j=Y, Z
$$

Simple comparison yields that $E\left[\Pi_{j}^{S}\right]$ is always greater than $E\left[\Pi_{j}^{N S}\right]$. Even though it is on average more profitable to behave strategically, the existence of profitable deviations induces the TV stations to behave non-strategically. When $v+\frac{1}{A}>\frac{1}{2}$, even an infinitesimally small value of the sampling cost gives rise to the non-strategic equilibrium.

When $v+c+\frac{1}{A} \leq \frac{1}{2}$, both equilibria can be supported as PBEs. However, as long as viewers rationally expect the TV stations to play the less costly strategies, the non-strategic 
equilibrium can be ruled out. To be more precise, provided that $v+c+\frac{1}{A} \leq \frac{1}{2}$, it is always optimal to play strategically for the TV stations when the viewers expect them to do so. However, if the viewers are pessimistic in the sense that they only expect the worse when they do not see a tune-in, the unique PBE is the non-strategic one.

\subsection{Social Value of Tune-ins}

In this section, I analyze the effects of a possible ban on the use of tune-ins. I compare the expected social welfare under a hypothetical "no tune-in" regime with that under no restrictions. In the Appendix, I find the expected utility of a random viewer in all of the possible three situations: the strategic equilibrium $(\mathrm{S})$, the non-strategic equilibrium (NS), and a "no tune-in" equilibrium (NT).

In a regime of no tune-ins, ex-ante expected per-viewer profit of a station in the second period is just the average of the audience shares given in Table 1, multiplied with the number of commercials and the per-viewer price. So, the total ex-ante expected per-viewer profits of a station are given by,

$$
E\left[\Pi_{j}^{N T}\right]=A\left[\frac{1}{2}+\frac{6(v+c)+1}{9}\right] p, \quad j=Y, Z
$$

Let $W$ denote the social welfare which is defined as the summation of station profits and viewer well-being. Then, the change in expected social welfare when the non-strategic PBE arises is expressed as,

$$
\begin{aligned}
E\left[W^{N S}-W^{N T}\right] & =N\left(E_{\lambda}\left[U_{\lambda}^{N S}-U_{\lambda}^{N T}\right]+2 E\left[\Pi_{j}^{N S}-\Pi_{j}^{N T}\right]\right) \\
& =N\left(\left(\frac{15}{2}-c-6 v\right) \frac{c}{9}-2\left(\frac{p}{3}+\frac{2 c A}{9}\right)\right)
\end{aligned}
$$

Note that $\left(\frac{15}{2}-c-6 v-4 A\right)<0$ by Assumption (1) (even a much smaller value of $A$ would imply the same result). So, $E\left[W^{N S}-W^{N T}\right]=N\left(\left(\frac{15}{2}-c-6 v-4 A\right) \frac{c}{9}-\frac{2 p}{3}\right)<0$ for all parameter values. This means that it is welfare improving to ban the use of tune-ins when $v+c+\frac{1}{A}>\frac{1}{2}$, since non-strategic equilibrium is the unique symmetric PBE for these parameter values. Although viewers are obviously better off when there are tune-ins, it may 
be the case that lost revenues are too high, and therefore it is better to ban tune-ins. The primary reason for why the stations lose that much revenue is that fewer people watch TV when there are more tune-ins in general. In the absence of a ban, the "no tune-in" regime is not sustainable as an equilibrium because of unilateral deviations.

The same result does not carry over to the strategic equilibrium. The reason is that the expected audience size in the strategic equilibrium is equal with that in the "no tunein" regime. Recalling that $E\left[\Pi_{j}^{S}\right]=\left[\frac{A}{2}+\frac{(6(v+c)+1) A}{9}-\frac{2}{9}\right] p$, the change in expected social welfare when the strategic PBE is the outcome is,

$$
\begin{aligned}
E\left[W^{S}-W^{N T}\right] & =N\left(E_{\lambda}\left[U_{\lambda}^{S}-U_{\lambda}^{N T}\right]+2 E\left[\Pi_{j}^{S}-\Pi_{j}^{N T}\right]\right) \\
& =N\left(\frac{c}{3}-\frac{4 p}{9}\right)
\end{aligned}
$$

which is negative when $p>\frac{3 c}{4}$.

Proposition 3 If the outcome is the strategic PBE, it is welfare improving to ban tune-ins only when $p>\frac{3 c}{4}$. If it is the non-strategic PBE, on the other hand, it is always welfare improving to ban tune-ins.

It immediately follows from Proposition (3) that it may be welfare improving if the two stations collude and maximize total advertisement revenues. It is optimal to air no tune-ins in such a case, and as long as the conditions of Proposition (3) hold, this is better for the society as a whole.

Tune-ins are clearly beneficial for viewers. Without tune-ins, viewers would engage in too much sampling and some of them would end up watching TV although this yields a negative utility. If viewers had complete information about program attributes, TV stations would serve to a smaller audience size. However, information is incomplete and it is not feasible to inform everyone about TV programs. In a non-strategic equilibrium, TV stations are forced by market forces to air too many tune-ins. This is due to two factors. First, an equilibrium with no tune-ins is not feasible because of the oligopoly structure; without tune-ins, more people would switch away. Second, strategic equilibrium is not credible when $c$ and/or $\frac{1}{A}$ is 
large. Had viewers believed that the strategic PBE would arise, neither station would have incentive to air any tune-ins at all. When $c$ is large, a station can still capture an audience size that is high enough to make it worthwhile to deviate. When $A$ is small, second-period revenue is not that large anyway, so deviation is again profitable. But, viewers are rational and perfectly anticipate these incentives beforehand. As a result, the stations are forced to air more tune-ins. The higher the number of tune-ins, the better choices people make, which implies a smaller audience size in the second period. So, the stations are double jeopardized when the strategic equilibrium cannot be attained; a higher number of tune-ins and fewer viewers on average. The revenue they lose in such a situation is larger than the increase in the well-being of viewers. Therefore, banning tune-ins is welfare enhancing.

The latter one of the two factors above is also present in the strategic equilibrium. However, since $c$ or $\frac{1}{A}$ is small enough, strategic equilibrium is credible. Therefore, the second factor does not arise. When the strategic equilibrium is attainable, stations do not end up airing too many tune-ins. Consumer surplus is now lower since viewers more often get stuck watching a program that is a bad match. When the per-viewer commercial price is low relative to the sampling cost, the decrease in the well-being of viewers is smaller than the increase in the revenues of the TV station due to fewer tune-ins, and therefore no intervention is necessary. ${ }^{6}$

\section{Discussion and Conclusion}

I have introduced a framework in which advertising decision of a firm provides information to people about the attributes of the product of the other firm in a horizontally differentiated duopoly market. I have chosen the TV industry for this analysis for several reasons. Most importantly, tune-ins are directly informative advertisements, they are special to the TV industry and they are exclusive (exclusive in the sense that a TV station cannot advertise

\footnotetext{
${ }^{6} 88.5$ million U.S. viewers watched the 2000 Super Bowl. The average price for a 30 -second commercial was $\$ 2.1$ million. So, the per-viewer price was approximately 2.4 cents. Although it is impossible to make an ordinal comparison of the per-viewer price and the sampling cost, common sense suggests that an average viewer loses more following an unsuccessful sampling.
} 
to other stations' audiences).

I have analyzed the provision of tune-ins in a two-period model of TV broadcasting where programs are provided by two TV stations and are only horizontally differentiated. For simplicity, I assumed that the first programs at each TV station are known to viewers beforehand, and that the market is covered in the first period. The locations of the second programs are ex-ante unknown to people, although they know that the TV stations have this information. Therefore, viewers know that the tune-in decision of the TV station they watch in the first period may provide them with information about the location of the other station's program. In this context, I have characterized the symmetric perfect Bayesian equilibria in which tune-in decisions of the stations and the following inferences of viewers are in accordance.

The main aim of the analysis is to characterize the nature of strategic behavior when TV stations are privately informed about both programs. Therefore, I have restricted the parameter values so as to ensure that an equilibrium in which no tune-ins are aired does not exist. These restrictions simply require that the number of non-program breaks is not very small, and that the value of the sampling cost is small relative to the baseline utility people derive when they watch their ideal programs. The latter one implies that viewers always choose to sample a station if their priors for the program at that station are unchanged. However, it is also implied that the baseline utility is not very large so that all of the viewers do not watch the second program until the end. I believe that these assumptions are in line with empirical regularities and do not impose restrictions on the findings.

Existence of two symmetric PBEs has been shown. In the first one - referred to as the strategic PBE in the text - each station's tune-in decision depends on the location of its own as well as the location of its rival's program. A station chooses not to air a tune-in whenever at least one of the programs is such that no first-period viewers of that station would watch it. Not airing a tune-in when a station's own program is not appealing to its first-period viewers is optimal because a tune-in would not bring in any viewers. Similarly, it is not optimal to air a tune-in when a station knows that its first-period viewers will not 
like the program at the other station, and therefore anyone who may initially switch to the other station will come back.

The second possible PBE - referred to as the non-strategic PBE in the text - is the one in which each station's tune-in decision only depends on the location of its own program. A station chooses not to air a tune-in only when its program is such that none of its first-period viewers would watch it. This PBE is shown to exist as long as Assumption (1) is satisfied, and to be the unique equilibrium when either the sampling cost is relatively high or the TV stations have a small number of non-program breaks (or both). In the opposite case, both equilibria are valid. It is at first ambigous as to which one of these equilibria would be selected. If viewers are extremely pessimistic, they would expect the worst scenario upon not seeing a tune-in. This belief structure would eliminate the strategic PBE. However, there is no reason for viewers to be pessimistic in the current model. Since they perfectly know the incentives of the TV stations, they would most likely anticipate that the TV stations would choose the strategies resulting in higher revenues. From their point of view, the expected revenues are higher in the strategic PBE since the stations air fewer tune-ins on average compared to the non-strategic PBE. So, there is not much reason to believe that the stations would behave non-strategically. If, however, viewers were exogenously assumed to be pessimistic, we would have had the non-strategic PBE as the unique PBE.

In the strategic PBE, viewers update their priors about the location of the other station's program upon observing the tune-in decision of the station they have watched in the first period. So, the tune-in decision of the station they have watched in the first period serves as a signal for the program of the other station. There does not exist a fully separating equilibrium in which viewers are able to locate the other station's program with certainty only based on the tune-in decision of a TV station. If a station does not air a tune-in, it is inferred by that station's first-period audience that the upcoming program is probably not a good match for them. This deters them away from staying at the same station. However, they also infer that it could actually be the other station's program that is a bad match. In the strategic PBE, this additional inference exactly offsets the deterrence effect. As a result, 
viewers are indifferent between choosing either one of the two stations to start watching. After learning the location of the program they chose to sample first, they update their beliefs about the other program and then make their final decisions. At some instances, perfect revelation may occur after sampling one of the stations. Suppose a viewer watches station $Y$ in the first period and station $Y$ does not air a tune-in for its upcoming program. If this viewer continues to watch station $Y$ and discovers after sampling that the program at $Y$ is appealing to her, then she perfectly infers the location of the program at the other station. Had the TV stations behaved non-strategically, this inference would be impossible to reach.

The stations are better off on average in the strategic PBE compared to the non-strategic one. The same is not true for the viewers; unsuccessful sampling occurs less frequently in the strategic PBE since TV stations air fewer tune-ins. From a welfare perspective, it may be desirable to ban tune-ins. In the non-strategic PBE, total revenues of the stations are significantly reduced compared to the strategic PBE and this reduction overweights the increase in the aggregate viewer surplus. The real life is clearly not as simple. It is almost impossible to represent TV programs in a one-dimensional space. It is also not likely that viewers' preferences stay the same over time, and that viewers have nonrandom utilities.Nevertheless, this paper raises a question that has been untouched before, and constitutes a starting point for a more thorough analysis of the welfare properties of tune-ins in a duopoly TV industry. 


\section{Appendix}

In this appendix, I find the utility of a random viewer under three specifications; the strategic PBE (S), the non-strategic PBE (NS), and the "no tune-in" regime (NT). I assume that the viewer located at $\frac{1}{2}$ watches station $Y$ in the first period, and that whenever a viewer is indifferent between staying at a station or sampling the other one (or switching off), she chooses to stay (these assumptions do not change the results since there is a continuum of viewers). The findings in this appendix help me calculate the social welfare in Section 4 . In each one of the nine possible program locations, average viewer derives a different level of utility since the TV stations, and in turn the viewers, behave differently in each one. Only the first five cases are analyzed in detail. The remaining four cases are symmetric with the first four ones.

Case (1): $(y, z)=(0,0)$.

S: Station $Y$ does, $Z$ does not air a tune-in. Among those who watched $Y$ in the first period, $\lambda \leq \frac{1}{4}$ stay with $Y$ while the others switch to $Z$ before the second period starts. After seeing that $z=0, \lambda>v+c$ switch off. The ones who watched $Z$ in the first period end up sampling both stations and eventually turn their TVs off. So,

$$
U_{\lambda}^{S}= \begin{cases}v-\lambda & \text {,if } 0 \leq \lambda \leq v+c \\ -c & \text {,if } v+c<\lambda \leq \frac{1}{2} \\ -2 c & \text {,if } \frac{1}{2}<\lambda \leq 1\end{cases}
$$

NS: Station $Y$ does, $Z$ does not air a tune-in. Among those who watched $Y$ in the first period, $\lambda \leq \frac{1}{4}+\frac{c}{2}$ stay with $Y$ after seeing a tune-in for $y=0$ while the others switch to $Z$. The ones who watched $Z$ in the first period only sample $Y$ since they infer that $z=0$. But they eventually turn their TVs off. So,

$$
U_{\lambda}^{N S}= \begin{cases}v-\lambda & \text {,if } 0 \leq \lambda \leq v+c \\ -c & \text {,if } v+c<\lambda \leq 1\end{cases}
$$

NT: A random half of viewers start with $Y$ and the other half with $Z$. Viewers with locations $\lambda \leq \frac{1}{4}+\frac{3 c}{2}$ settle in the first station they sampled, thus incurring no sampling cost, while those with $\frac{1}{4}+\frac{3 c}{2} \leq \lambda<v+c$ sample both stations and choose one at random. All others 
switch off after sampling both stations. So,

$$
U_{\lambda}^{N T}= \begin{cases}v-\lambda & \text {,if } 0 \leq \lambda \leq \frac{1}{4}+\frac{3 c}{2} \\ v-c-\lambda & , \text { if } \frac{1}{4}+\frac{3 c}{2}<\lambda \leq v+c \\ -2 c & \text {,if } v+c<\lambda \leq 1\end{cases}
$$

So, we have the following:

$$
\begin{gathered}
U_{\lambda}^{S}-U_{\lambda}^{N T}= \begin{cases}0 & \text {,if } 0 \leq \lambda \leq \frac{1}{4}+\frac{3 c}{2} \\
c & \text {,if } \frac{1}{4}+\frac{3 c}{2}<\lambda \leq \frac{1}{2} \\
0 & \text {,if } \frac{1}{2}<\lambda \leq 1\end{cases} \\
U_{\lambda}^{N S}-U_{\lambda}^{N T}= \begin{cases}0 & \text {,if } 0 \leq \lambda \leq \frac{1}{4}+\frac{3 c}{2} \\
c & \text {,if } \frac{1}{4}+\frac{3 c}{2}<\lambda \leq 1\end{cases}
\end{gathered}
$$

Integrating over $\lambda$, we get:

$$
\begin{aligned}
E_{\lambda}\left[U_{\lambda}^{S}-U_{\lambda}^{N T} \mid(y, z)=(0,0)\right] & =\left(\frac{1}{4}-\frac{3 c}{2}\right) c \\
E_{\lambda}\left[U_{\lambda}^{N S}-U_{\lambda}^{N T} \mid(y, z)\right. & =(0,0)]=\left(\frac{3}{4}-\frac{3 c}{2}\right) c
\end{aligned}
$$

Case (2): $(y, z)=\left(0, \frac{1}{2}\right)$.

S: Station $Y$ does, $Z$ does not air a tune-in. Among those who watched $Y$ in the first period, $\lambda \leq \frac{1}{4}$ stay with $Y$ while the others switch to $Z$ and stay there. Among those who watched $Z$ in the first period, a random half stay with $Z$. After seeing that $z=\frac{1}{2}$, they infer that $y=0$, so $\frac{1}{2} \leq \lambda \leq \frac{1}{2}+v+c$ stay and the others switch off. The other half start sampling with $Y$. After seeing that $y=0$, they infer $z \in\left\{0, \frac{1}{2}, 1\right\}$, so all switch to $Z$. Those with $\frac{1}{2} \leq \lambda \leq \frac{1}{2}+v+c$ stay, the others switch off. So,

$$
U_{\lambda}^{S}= \begin{cases}v-\lambda & \text {,if } 0 \leq \lambda \leq \frac{1}{4} \\ v-\left(\frac{1}{2}-\lambda\right) & \text {,if } \frac{1}{4}<\lambda \leq \frac{1}{2} \\ \frac{1}{2}\left(v-\lambda+\frac{1}{2}\right)+\frac{1}{2}\left(v-c-\lambda+\frac{1}{2}\right) & \text {,if } \frac{1}{2}<\lambda \leq \frac{1}{2}+v+c \\ \frac{1}{2}(-c)+\frac{1}{2}(-2 c) & \text {,if } \frac{1}{2}+v+c<\lambda \leq 1\end{cases}
$$

NS: Both stations air a tune-in. $\lambda \leq \frac{1}{4}+\frac{c}{2}$ among those who watched $Y$ in the first period stay with $Y$ after seeing a tune-in for $y=0$ while the others switch to $Z$ and stay there. Behavior of the ones who watched $Z$ in the first period is similar. Those with $\lambda>\frac{3}{4}+\frac{c}{2}$ initially 
switch to $Y$ in the hope of finding out $y=1$. After discovering that $y=0, \frac{3}{4}+\frac{c}{2}<\lambda \leq \frac{1}{2}+v$ come back to $Z$ while the others turn their TVs off. So,

$$
U_{\lambda}^{N S}= \begin{cases}v-\lambda & \text {,if } 0 \leq \lambda \leq \frac{1}{4}+\frac{c}{2} \\ v-\left(\frac{1}{2}-\lambda\right) & \text {,if } \frac{1}{4}+\frac{c}{2}<\lambda \leq \frac{1}{2} \\ v-\left(\lambda-\frac{1}{2}\right) & \text {,if } \frac{1}{2}<\lambda \leq \frac{3}{4}+\frac{c}{2} \\ v-c-\left(\lambda-\frac{1}{2}\right) & \text {,if } \frac{3}{4}+\frac{c}{2}<\lambda \leq \frac{1}{2}+v \\ -c & \text {,if } \frac{1}{2}+v<\lambda \leq 1\end{cases}
$$

NT: A random half of viewers start with $Y$ and the other half with $Z$. Viewers with locations $\frac{1}{4}-\frac{3 c}{2} \leq \lambda \leq \frac{1}{4}+\frac{3 c}{2}$ settle in the first station they sampled, thus incurring no sampling cost, while the others may end up sampling both stations. For $\lambda \leq \frac{1}{4}-\frac{3 c}{2}$, if the viewer is lucky and started with $Y$, she stays there. If she started with $Z$, she also samples $Y$. Similarly, $\frac{1}{4}+\frac{3 c}{2} \leq \lambda \leq \frac{3}{4}+\frac{3 c}{2}$ end up at $Z$ either immediately or after initially sampling $Y$. All others sample both stations and those with $\frac{3}{4}+\frac{3 c}{2} \leq \lambda \leq \frac{1}{2}+v+c$ stay tuned. So,

$$
U_{\lambda}^{N T}= \begin{cases}\frac{1}{2}(v-\lambda)+\frac{1}{2}(v-c-\lambda) & \text {,if } 0 \leq \lambda<\frac{1}{4}-\frac{3 c}{2} \\ \frac{1}{2}(v-\lambda)+\frac{1}{2}\left(v-\frac{1}{2}+\lambda\right) & \text {,if } \frac{1}{4}-\frac{3 c}{2} \leq \lambda \leq \frac{1}{4}+\frac{3 c}{2} \\ \frac{1}{2}\left(v-\frac{1}{2}+\lambda\right)+\frac{1}{2}\left(v-c-\frac{1}{2}+\lambda\right) & \text {,if } \frac{1}{4}+\frac{3 c}{2}<\lambda \leq \frac{1}{2} \\ \frac{1}{2}\left(v-\lambda+\frac{1}{2}\right)+\frac{1}{2}\left(v-c-\lambda+\frac{1}{2}\right) & \text {,if } \frac{1}{2}<\lambda \leq \frac{3}{4}+\frac{3 c}{2} \\ v-c-\left(\lambda-\frac{1}{2}\right) & \text {,if } \frac{3}{4}+\frac{3 c}{2}<\lambda \leq \frac{1}{2}+v+c \\ -2 c & \text {,if } \frac{1}{2}+v+c<\lambda \leq 1\end{cases}
$$

So, we have the following:

$$
\begin{gathered}
U_{\lambda}^{S}-U_{\lambda}^{N T}= \begin{cases}\frac{c}{2} & \text {,if } 0 \leq \lambda<\frac{1}{4}-\frac{3 c}{2} \\
\frac{1}{4}-\lambda & \text {,if } \frac{1}{4}-\frac{3 c}{2} \leq \lambda \leq \frac{1}{4} \\
\lambda-\frac{1}{4} & \text {,if } \frac{1}{4}<\lambda \leq \frac{1}{4}+\frac{3 c}{2} \\
\frac{c}{2} & \text {,if } \frac{1}{4}+\frac{3 c}{2}<\lambda \leq \frac{1}{2} \\
0 & \text {,if } \frac{1}{2}<\lambda \leq \frac{3}{4}+\frac{3 c}{2} \\
\frac{c}{2} & \text {,if } \frac{3}{4}+\frac{3 c}{2}<\lambda \leq 1\end{cases} \\
U_{\lambda}^{N S}-U_{\lambda}^{N T}= \begin{cases}\frac{c}{2} & \text {,if } 0 \leq \lambda<\frac{1}{4}-\frac{3 c}{2} \\
\frac{1}{4}-\lambda & \text {,if } \frac{1}{4}-\frac{3 c}{2} \leq \lambda \leq \frac{1}{4}+\frac{c}{2} \\
\lambda-\frac{1}{4} & \text {,if } \frac{1}{4}+\frac{c}{2}<\lambda \leq \frac{1}{4}+\frac{3 c}{2} \\
\frac{c}{2} & \text {,if } \frac{1}{4}+\frac{3 c}{2}<\lambda \leq \frac{3}{4}+\frac{c}{2} \\
-\frac{c}{2} & \text {,if } \frac{3}{4}+\frac{c}{2}<\lambda \leq \frac{3}{4}+\frac{3 c}{2} \\
0 & \text {,if } \frac{3}{4}+\frac{3 c}{2}<\lambda \leq \frac{1}{2}+v \\
\left(\lambda-\frac{1}{2}\right)-v & \text {,if } \frac{1}{2}+v<\lambda \leq \frac{1}{2}+v+c \\
c & \text {,if } \frac{1}{2}+v+c<\lambda \leq 1\end{cases}
\end{gathered}
$$


Integrating over $\lambda$, we get:

$$
\begin{aligned}
E_{\lambda}\left[U_{\lambda}^{S}-U_{\lambda}^{N T} \mid(y, z)=\left(0, \frac{1}{2}\right)\right] & =\left(\frac{3}{4}-\frac{9 c}{2}\right) \frac{c}{2}+\frac{9 c^{2}}{2}=\left(\frac{3}{8}+\frac{9 c}{4}\right) c \\
E_{\lambda}\left[U_{\lambda}^{N S}-U_{\lambda}^{N T} \mid(y, z)\right. & \left.=\left(0, \frac{1}{2}\right)\right]=\left(\frac{3}{4}-\frac{5 c}{2}\right) \frac{c}{2}+4 c^{2}+\left(\frac{1}{2}-v\right) c=\left(\frac{7}{8}+\frac{9 c}{4}-v\right) c
\end{aligned}
$$

Case (3): $(y, z)=(0,1)$.

S: Neither station airs a tune-in. Among those who watched $Y$ in the first period, a random half stay with $Y$ and infer that $z=1$ after seeing $y=0$. So, $0 \leq \lambda \leq v+c$ stay and the others switch off. The other half initially switch to $Z$. All of these viewers also sample $Y$ after discovering that $z=1$ and $0 \leq \lambda \leq v+c$ stay. Behavior of the viewers who watched $Z$ in the first period is just symmetric. So,

$$
U_{\lambda}^{S}= \begin{cases}\frac{1}{2}(v-\lambda)+\frac{1}{2}(v-c-\lambda) & \text {,if } 0 \leq \lambda \leq v+c \\ \frac{1}{2}(-c)+\frac{1}{2}(-2 c) & \text {,if } v+c<\lambda<1-(v+c) \\ \frac{1}{2}(v-1+\lambda)+\frac{1}{2}(v-c-1+\lambda) & \text {,if } 1-(v+c) \leq \lambda \leq 1\end{cases}
$$

NS: Both stations air a tune-in. So, $\lambda \leq \frac{1}{4}+\frac{c}{2}$ continue to stay with $Y$ while $\frac{1}{4}+\frac{c}{2}<\lambda \leq v$ come back to $Y$ after initially sampling $Z$. Behavior of the viewers who watched $Z$ in the first period is just symmetric. So,

$$
U_{\lambda}^{N S}= \begin{cases}v-\lambda & \text {,if } 0 \leq \lambda \leq \frac{1}{4}+\frac{c}{2} \\ v-c-\lambda & \text {,if } \frac{1}{4}+\frac{c}{2}<\lambda \leq v \\ -c & \text {,if } v<\lambda<1-v \\ v-c-(1-\lambda) & \text {,if } 1-v \leq \lambda<\frac{3}{4}-\frac{c}{2} \\ v-(1-\lambda) & \text {,if } \frac{3}{4}-\frac{c}{2} \leq \lambda \leq 1\end{cases}
$$

NT: The viewing choices here are similar with the previous case.

$$
U_{\lambda}^{N T}= \begin{cases}\frac{1}{2}(v-\lambda)+\frac{1}{2}(v-c-\lambda) & \text {,if } 0 \leq \lambda \leq \frac{1}{4}+\frac{3 c}{2} \\ v-c-\lambda & \text {,if } \frac{1}{4}+\frac{3 c}{2}<\lambda \leq v+c \\ -2 c & \text {,if } v+c<\lambda<1-(v+c) \\ v-c-(1-\lambda) & \text {,if } 1-(v+c) \leq \lambda<\frac{3}{4}-\frac{3 c}{2} \\ \frac{1}{2}(v-1+\lambda)+\frac{1}{2}(v-c-1+\lambda) & \text {,if } \frac{3}{4}-\frac{3 c}{2} \leq \lambda \leq 1\end{cases}
$$

So, we have the following:

$$
U_{\lambda}^{S}-U_{\lambda}^{N T}= \begin{cases}0 & \text {,if } 0 \leq \lambda \leq \frac{1}{4}+\frac{3 c}{2} \\ \frac{c}{2} & \text {,if } \frac{1}{4}+\frac{3 c}{2} \leq \lambda<\frac{3}{4}-\frac{3 c}{2} \\ 0 & \text {,if } \frac{3}{4}-\frac{3 c}{2} \leq \lambda \leq 1\end{cases}
$$




$$
U_{\lambda}^{N S}-U_{\lambda}^{N T}= \begin{cases}\frac{c}{2} & \text {,if } 0 \leq \lambda \leq \frac{1}{4}+\frac{c}{2} \\ -\frac{c}{2} & , \text { if } \frac{1}{4}+\frac{c}{2}<\lambda \leq \frac{1}{4}+\frac{3 c}{2} \\ 0 & , \text { if } \frac{1}{4}+\frac{3 c}{2}<\lambda \leq v \\ \lambda-v & , \text { if } v<\lambda \leq v+c \\ c & , \text { if } v+c<\lambda<1-(v+c) \\ 1-\lambda-v & , \text { if } 1-(v+c)<\lambda<1-v \\ 0 & , \text { if } 1-v<\lambda<\frac{3}{4}-\frac{3 c}{2} \\ -\frac{c}{2} & , \text { if } \frac{3}{4}-\frac{3 c}{2} \leq \lambda<\frac{3}{4}-\frac{c}{2} \\ \frac{c}{2} & , \text { if } \frac{3}{4}-\frac{c}{2} \leq \lambda \leq 1\end{cases}
$$

Integrating over $\lambda$, we get:

$$
\begin{aligned}
E_{\lambda}\left[U_{\lambda}^{S}-U_{\lambda}^{N T} \mid(y, z)=(0,1)\right] & =\left(\frac{1}{4}-\frac{3 c}{2}\right) c \\
E_{\lambda}\left[U_{\lambda}^{N S}-U_{\lambda}^{N T} \mid(y, z)\right. & =(0,1)]=\left(\frac{5}{4}-\frac{3 c}{2}-2 v\right) c
\end{aligned}
$$

Case $(4):(y, z)=\left(\frac{1}{2}, 0\right)$.

S: Station $Y$ does, $Z$ does not air a tune-in. Among those who watched $Y$ in the first period, $\lambda \geq \frac{1}{4}$ stay with $Y$ while the others initially switch to $Z$ and stay there after seeing that $z=0$. Among those who watched $Z$ in the first period, the random half that started sampling with $Y$ are lucky as they infer that $z=0$. So, those with $\frac{1}{2} \leq \lambda \leq \frac{1}{2}+v+c$ stay, the others turn their TVs off. The other half sample both stations and those with $\frac{1}{2} \leq \lambda \leq \frac{1}{2}+v+c$ end up watching $Y$. So,

$$
U_{\lambda}^{S}= \begin{cases}v-\lambda & \text {,if } 0 \leq \lambda<\frac{1}{4} \\ v-\left(\frac{1}{2}-\lambda\right) & \text {,if } \frac{1}{4} \leq \lambda \leq \frac{1}{2} \\ \frac{1}{2}\left(v-\lambda+\frac{1}{2}\right)+\frac{1}{2}\left(v-c-\lambda+\frac{1}{2}\right) & \text {,if } \frac{1}{2}<\lambda \leq \frac{1}{2}+v+c \\ \frac{1}{2}(-c)+\frac{1}{2}(-2 c) & \text {,if } \frac{1}{2}+v+c<\lambda \leq 1\end{cases}
$$

NS: Station $Y$ does, $Z$ does not air a tune-in. Among those who watched $Y$ in the first period, $\lambda \geq \frac{1}{4}-\frac{c}{2}$ stay with $Y$ while the others initially switch to $Z$ and stay there after seeing that $z=0$. The viewers who watched $Z$ in the first period switch to $Y$ and those with $\frac{1}{2} \leq \lambda \leq \frac{1}{2}+v+c$ stay there. So,

$$
U_{\lambda}^{N S}= \begin{cases}v-\lambda & \text {,if } 0 \leq \lambda<\frac{1}{4}-\frac{c}{2} \\ v-\left(\frac{1}{2}-\lambda\right) & \text {,if } \frac{1}{4}-\frac{c}{2} \leq \lambda \leq \frac{1}{2} \\ v-\left(\lambda-\frac{1}{2}\right) & \text {,if } \frac{1}{2}<\lambda \leq \frac{1}{2}+v+c \\ -c & \text {,if } \frac{1}{2}+v+c<\lambda \leq 1\end{cases}
$$


NT: Same with Case(2). So,

$$
U_{\lambda}^{N T}= \begin{cases}\frac{1}{2}(v-\lambda)+\frac{1}{2}(v-c-\lambda) & \text {,if } 0 \leq \lambda<\frac{1}{4}-\frac{3 c}{2} \\ \frac{1}{2}(v-\lambda)+\frac{1}{2}\left(v-\frac{1}{2}+\lambda\right) & \text {,if } \frac{1}{4}-\frac{3 c}{2} \leq \lambda \leq \frac{1}{4}+\frac{3 c}{2} \\ \frac{1}{2}\left(v-\frac{1}{2}+\lambda\right)+\frac{1}{2}\left(v-c-\frac{1}{2}+\lambda\right) & \text {,if } \frac{1}{4}+\frac{3 c}{2}<\lambda \leq \frac{1}{2} \\ \frac{1}{2}\left(v-\lambda+\frac{1}{2}\right)+\frac{1}{2}\left(v-c-\lambda+\frac{1}{2}\right) & \text {,if } \frac{1}{2}<\lambda \leq \frac{3}{4}+\frac{3 c}{2} \\ v-c-\left(\lambda-\frac{1}{2}\right) & \text {,if } \frac{3}{4}+\frac{3 c}{2}<\lambda \leq \frac{1}{2}+v+c \\ -2 c & \text {,if } \frac{1}{2}+v+c<\lambda \leq 1\end{cases}
$$

So, we have the following:

$$
\begin{gathered}
U_{\lambda}^{S}-U_{\lambda}^{N T}= \begin{cases}\frac{c}{2} & \text {,if } 0 \leq \lambda<\frac{1}{4}-\frac{3 c}{2} \\
\frac{1}{4}-\lambda & \text {,if } \frac{1}{4}-\frac{3 c}{2} \leq \lambda<\frac{1}{4} \\
\lambda-\frac{1}{4} & \text {,if } \frac{1}{4} \leq \lambda \leq \frac{1}{4}+\frac{3 c}{2} \\
\frac{c}{2} & \text {,if } \frac{1}{4}+\frac{3 c}{2}<\lambda \leq \frac{1}{2} \\
0 & \text {,if } \frac{1}{2}<\lambda \leq \frac{3}{4}+\frac{3 c}{2} \\
\frac{c}{2} & \text {,if } \frac{3}{4}+\frac{3 c}{2}<\lambda \leq 1\end{cases} \\
U_{\lambda}^{N S}-U_{\lambda}^{N T}= \begin{cases}\frac{c}{2} & \text {,if } 0 \leq \lambda<\frac{1}{4}-\frac{3 c}{2} \\
\frac{1}{4}-\lambda & \text {,if } \frac{1}{4}-\frac{3 c}{2} \leq \lambda<\frac{1}{4}-\frac{c}{2} \\
\lambda-\frac{1}{4} & \text {,if } \frac{1}{4}-\frac{c}{2} \leq \lambda<\frac{1}{4}+\frac{3 c}{2} \\
\frac{c}{2} & \text {,if } \frac{1}{4}+\frac{3 c}{2}<\lambda \leq \frac{3}{4}+\frac{3 c}{2} \\
c & \text {,if } \frac{3}{4}+\frac{3 c}{2}<\lambda \leq 1\end{cases}
\end{gathered}
$$

Integrating over $\lambda$, we get:

$$
\begin{gathered}
E_{\lambda}\left[U_{\lambda}^{S}-U_{\lambda}^{N T} \mid(y, z)=\left(\frac{1}{2}, 0\right)\right]=\left(\frac{3}{4}-\frac{9 c}{2}\right) \frac{c}{2}+\frac{9 c^{2}}{2}=\left(\frac{3}{8}+\frac{9 c}{4}\right) c \\
E_{\lambda}\left[U_{\lambda}^{N S}-U_{\lambda}^{N T} \mid(y, z)=\left(\frac{1}{2}, 0\right)\right]=\left(\frac{5}{4}-\frac{9 c}{2}\right) \frac{c}{2}+4 c^{2}=\left(\frac{5}{8}+\frac{7 c}{4}\right) c
\end{gathered}
$$

Case (5): $(y, z)=\left(\frac{1}{2}, \frac{1}{2}\right)$.

S: Both stations air a tune-in. $\frac{1}{4} \leq \lambda \leq \frac{3}{4}$ stay with the stations they watched in the in the first period. The others initially switch to the other station; those with $\lambda<\frac{1}{2}-v-c$ and $\lambda>\frac{1}{2}+v+c$ switch off while the others stay. So,

$$
U_{\lambda}^{S}= \begin{cases}-c & \text { if } 0 \leq \lambda<\frac{1}{2}-v-c \\ v-\left(\frac{1}{2}-\lambda\right) & \text {,if } \frac{1}{2}-v-c \leq \lambda \leq \frac{1}{2} \\ v-\left(\lambda-\frac{1}{2}\right) & \text {,if } \frac{1}{2}<\lambda \leq \frac{1}{2}+v+c \\ -c & \text {,if } \frac{1}{2}+v+c<\lambda \leq 1\end{cases}
$$


NS: Both stations air a tune-in. $\frac{1}{4}-\frac{c}{2} \leq \lambda \leq \frac{3}{4}+\frac{c}{2}$ stay with the stations they watched in the in the first period. The others initially switch to the other station and stay there except for $\lambda<\frac{1}{2}-v-c$ and $\lambda>\frac{1}{2}+v+c$. So, $U_{\lambda}^{N S}$ remains the same as above.

NT: Those with $\frac{1}{4}-\frac{3 c}{2} \leq \lambda \leq \frac{3}{4}+\frac{3 c}{2}$ stay with the stations they sample first. Others sample both stations and those with $\frac{1}{2}-v-c \leq \lambda \leq \frac{1}{4}-\frac{3 c}{2}$ and $\frac{3}{4}+\frac{3 c}{2} \leq \lambda \leq \frac{1}{2}+v+c$ choose to watch one of them at random. The others turn their TVs off. So,

$$
U_{\lambda}^{N T}= \begin{cases}-2 c & \text { if } 0 \leq \lambda<\frac{1}{2}-v-c \\ v-c-\left(\frac{1}{2}-\lambda\right) & \text {,if } \frac{1}{2}-v-c \leq \lambda<\frac{1}{4}-\frac{3 c}{2} \\ v-\left(\frac{1}{2}-\lambda\right) & , \text { if } \frac{1}{4}-\frac{3 c}{2} \leq \lambda \leq \frac{1}{2} \\ v-\left(\lambda-\frac{1}{2}\right) & \text {,if } \frac{1}{2}<\lambda \leq \frac{3}{4}+\frac{3 c}{2} \\ v-c-\left(\lambda-\frac{1}{2}\right) & \text {,if } \frac{3}{4}+\frac{3 c}{2}<\lambda \leq \frac{1}{2}+v+c \\ -2 c & , \text { if } \frac{1}{2}+v+c<\lambda \leq 1\end{cases}
$$

So, we have the following:

$$
U_{\lambda}^{S}-U_{\lambda}^{N T}=U_{\lambda}^{N S}-U_{\lambda}^{N T}= \begin{cases}c & \text {,if } 0 \leq \lambda<\frac{1}{4}-\frac{3 c}{2} \\ 0 & \text {,if } \frac{1}{4}-\frac{3 c}{2} \leq \lambda \leq \frac{3}{4}+\frac{3 c}{2} \\ c & \text {,if } \frac{3}{4}+\frac{3 c}{2}<\lambda \leq 1\end{cases}
$$

Integrating over $\lambda$, we get:

$$
E_{\lambda}\left[U_{\lambda}^{S}-U_{\lambda}^{N T} \mid(y, z)=\left(\frac{1}{2}, \frac{1}{2}\right)\right]=E_{\lambda}\left[U_{\lambda}^{N S}-U_{\lambda}^{N T} \mid(y, z)=\left(\frac{1}{2}, \frac{1}{2}\right)\right]=\left(\frac{1}{2}-3 c\right) c
$$

The remaining four cases are symmetric with the first four cases, and therefore are omitted. Finally, integrating over $(y, z)$, we get:

$$
\begin{aligned}
E_{\lambda}\left[U_{\lambda}^{S}-U_{\lambda}^{N T}\right] & =\frac{1}{9}(3 c) \\
E_{\lambda}\left[U_{\lambda}^{N S}-U_{\lambda}^{N T}\right] & =\frac{1}{9}\left(\frac{15}{2}-6 v-c\right) c
\end{aligned}
$$




\section{References}

[1] Anand, Bharat N. and Ron Shachar (1998), "The Effectiveness and Targeting of Television Advertising," Journal of Economics and Management Strategy, 7, 363-396.

[2] Anand, Bharat N. and Ron Shachar (2006), "Targeted Advertising as a Signal," mimeo, Harvard Business School and Tel Aviv University.

[3] Anand, Bharat N. and Ron Shachar (2005), "Advertising the Matchmaker," mimeo, Harvard Business School and Tel Aviv University.

[4] Anderson, Simon P. and André de Palma (2006), "Information Congestion," mimeo, Université de Cergy-Pontoise and University of Virginia.

[5] Anderson, Simon P., André de Palma and Jacques-F. Thisse (1992), Discrete Choice Theory of Product Differentiation, MIT Press, Cambridge.

[6] Anderson, Simon P. and Régis Renault (2006), "Advertising Content," American Economic Review, 96, 1, 93-113.

[7] Anderson, Simon P. and Stephen Coate (2005), "Market Provision of Broadcasting: A Welfare Analysis," Review of Economic Studies, 72, 4, 947-972.

[8] Bagwell, Kyle. (2005) "The Economic Analysis of Advertising", working paper, Columbia University, forthcoming in M. Armstrong and R. Porter (eds.) Handbook of Industrial Organization, Vol. 3, North-Holland: Amsterdam.

[9] Butters, Gerard R. (1977), "Equilibrium Distributions of Sales and Advertising Prices," Review of Economic Studies, 44, 465-491.

[10] Çelik, Levent (2006), "Provision of Informative Advertising: The Case of Broadcasting," Chapter 2 of Doctoral Dissertation, University of Virginia.

[11] Crawford, Vincent P. and Joel Sobel (1982), "Strategic Information Transmission," Econometrica, 50, 6, 1431-1451. 
[12] De Bijl, Paul W. J. (1997) "Entry Deterrence and Signaling in Markets for Search Goods," International Journal of Industrial Organization, 16, 1-19.

[13] Diamond, Peter A. (1971), "A Model of Price Adjustment," Journal of Economic Theory, 3, 156-168.

[14] Dixit, Avinash K. and Victor D. Norman (1978), "Advertising and Welfare," Bell Journal of Economics, 9, 1-17.

[15] Dukes, Anthony (2004), "The Advertising Market in a Product Oligopoly," Journal of Industrial Economics, 52, 327-348.

[16] Fluet, Claude and Paolo G. Garella (2002), "Advertising and Prices as Signals of Quality in a Regime of Price Rivalry," International Journal of Industrial Organization, 20, 907930.

[17] Gabszewicz, Jean, Didier Laussel and Nathalie Sonnac (2004), "Programming and Advertising Competition in the Broadcasting Industry," Journal of Economics 85 Management Strategy, 13 (4), 657-669.

[18] Grossman, Gene and Carl Shapiro (1984), "Informative Advertising with Differentiated Products," Review of Economic Studies, 51, 63-81.

[19] Hertzendorf, Mark N. and Per Baltzer Overgaard (2001), "Price Competition and Advertising Signals: Signaling by Competing Senders," Journal of Economics and Management Strategy, 10, 4, 621-663.

[20] Hertzendorf, Mark N. and Per Baltzer Overgaard (2002), "Prices as Signals of Quality in Duopoly,", mimeo, University of Aarhus.

[21] Kihlstrom, Richard E. and Michael H. Riordan (1984), "Advertising as a Signal," Journal of Political Economy, 92, 427-450. 
[22] Matthews, Steven A. and Doron Fertig (1990), "Advertising Signals of Product Quality," Discussion Paper No. 881, Northwestern University.

[23] Meurer, Michael and Dale O. Stahl, II (1994), "Informative Advertising and Product Match," International Journal of Industrial Organization, 12, 1-19.

[24] Milgrom, Paul R. (1981), "Good News and Bad News: Representation Theorems and Applications," Bell Journal of Economics, 12, 380-391.

[25] Milgrom, Paul R. and John M. Roberts (1986a), "Prices and Advertising as Signals of Product Quality," Journal of Political Economy, 94, 796-821.

[26] Milgrom, Paul R. and John M. Roberts (1986b), "Relying on the Information of Interested Parties," Rand Journal of Economics, 17, 1, 18-32.

[27] Moraga-González, José Luis (2000), "Quality Uncertainty and Informative Advertising," International Journal of Industrial Organization, 18, 615-640.

[28] Nelson, Phillip J. (1974), "Advertising as Information," Journal of Political Economy, $82,729-754$.

[29] Roberts, Jacques and Dale O. Stahl, II (1993), "Informative Price Advertising in a Sequential Search Model," Econometrica, 61 (3), 657-686.

[30] Shapiro, Carl (1980), "Advertising and Welfare: Comment," Bell Journal of Economics, $11,749-752$.

[31] Tirole, Jean (1998), The Theory of Industrial Organization, MIT Press, Cambridge.

[32] Wolinsky, Asher (1984), "Product Differentiation with Imperfect Information," Review of Economic Studies, 51, 53-61. 